\title{
Relationship Between Social Support and Psychological Resilience of Patients with Convalescent Stroke: Mediating Effect of Self-Efficacy
}

\author{
Weiwei Bai ${ }^{1}$, Yanmei Liu',,*, Meng Li ${ }^{1}$, Yueli Hou ${ }^{1}$, Shaohua Lei ${ }^{1}$ \\ ${ }^{1}$ School of Nursing, Shaanxi University of Chinese Medicine, Xianyang 712046, China. \\ ${ }^{2}$ Shaanxi Province Hospital of Traditional Chinese Medicine, Xi'an 710003, China.
}

\begin{abstract}
How to cite this paper: Weiwei Bai, Yanmei Liu, Meng Li, Yueli Hou, Shaohua Lei. (2020) Relationship Between Social Support and Psychological Resilience of Patients with Convalescent Stroke: Mediating Effect of Self-Efficacy. International Journal of Clinical and Experimental Medicine Research, 4(2), 21-27.

DOI: 10.26855/ijcemr.2020.04.004
\end{abstract}

Received: March 22, 2020

Accepted: April 25, 2020

Published: May 7, 2020

*Corresponding author: Yanmei Liu, Shaanxi Province Hospital of Traditional Chinese Medicine, Xi'an 710003, China.

Email:1305999760@qq.com

\begin{abstract}
Objectives: To explore the relationship among psychological resilience, social support and self-efficacy of patients with convalescent stroke. Methods: From May 1 to December 31 in 2019, a total of 288 patients with convalescent stroke were investigated with the Social Support Rating Scale(SSRS), Stroke self-efficacy questionnaire (SSEQ), Connor-Davidson resilience scale (CD-RISC) and the general information questionnaire. Correlation analysis and mediating effect analysis were done. Results: The scores of social support, self-efficacy, psychological resilience are (34.25 \pm 5.95$),(60.28 \pm 13.11)$ and $(61.90 \pm 13.84)$, respectively. Psychological resilience is shown to be significantly correlated with social support $(r=0.778, P<0.01)$ and self-efficacy $(r=0.750, P<0.01)$. Self-efficacy had an incomplete mediating effect between social support and psychological resilience, and the mediation ratio was $53.30 \%$. Conclusions: There is a positive correlation between social support, self-efficacy and psychological resilience in patients with convalescent stroke. Self-efficacy plays a part of mediating role between social support and psychological resilience.
\end{abstract}

\section{Keywords}

Stroke, Social Support, Self-efficacy, Psychological Resilience

\section{Introduction}

The World Stroke Organization (WSO) released a report in 2019 globally showing currently more than 80 million stroke patients, and more than 13.7 million new stroke patients occur each year [1]. In China, $80 \%$ of stroke patients have serious sequelae, i.e., physical activity disorder, unclear speech, etc, and the disability rate is as high as $75 \%$. As a result, the self-care ability of some patients is constantly reduced or even lost $[2,3]$. Psychological resilience is a positive quality in personality traits. A higher level of psychological resilience is conducive to improving the ability of patients to solve problems and avoid fear, sadness or other bad emotions [4]. Social support can providing spiritual and material support can not only improve the individual's coping ability, but also effectively reduce the negative impact of stressful events on individuals. Self-efficacy is a key factor for 
stroke patients to face traumatic events and adverse outcomes [5]. This study analyzed the relationship among psychological resilience, social support and self-efficacy of patients with convalescent stroke, which helps clinical medical staff pay attention to the physiological and psychological health needs of patients with convalescent stroke and take corresponding nursing intervention measures, so as to improve the physical and mental health of patients.

\section{Objects and Methods}

\subsection{Objects of Study}

The study used a convenient sampling method. Patients were recruited by Shaanxi Province Hospital of traditional Chinese medicine, from May 1 to December 31 in 2019. Inclusion criteria were: (1) refer to the diagnostic criteria of the fourth national cerebrovascular disease, and brain magnetic resonance imaging (MRI) or computed tomography (CT) confirmed as cerebral apoplexy [6]; (2) age of $\geq 18$ years; (3) clear consciousness and patients can use language or text communication; (4) duration of disease within 2 weeks to 6 months. Exclusion criteria were: (1) comorbid major diseases, such as malignant tumors; (2) persons with a history of mental illness or undergoing psychotherapy; (3) blind, deaf, aphasia patients; (4) participate in other research projects at the same time. Informed consent was obtained from the patients who agreed to participate in the study. This study is approved by the Shaanxi Province Hospital of traditional Chinese medicine Institutional Review Board.

\subsection{Methods}

\subsubsection{Research tools}

(1) General information questionnaire

The general information questionnaire is mainly used to investigate the general demographic data and analyze the basic information of patients with convalescent stroke. Including gender, age, marital status, education level, type of stroke and stroke severity, etc. The severity of stroke is assessed by the Modified Barthel Index (MBI) scale, which included 10 items with a total score of 100 . The lower the score is, the more severe the disability is. $\mathrm{MBI}<95$ is defined as post-stroke disability, where $\mathrm{MBI} \leq 20$ is classified as complete disability, 21 40 is severe disability, $41 \sim 60$ is moderate disability, 61 94 is mild disability. While MBI $\geq 95$ is classified as without disability means that the daily self-care ability is completely independent. In the current study, the Cronbach's $\alpha$ values was 0.870 with good reliability.

(2) Connor-Davidson resilience scale (CD-RISC)

The CD-RISC developed by Dr Connor and Dr Davidson [7] and was translated in China by Yu [8], containing 25 items and three dimensions, which are the tenacity dimension, the self-improvement dimension and the optimistic dimension respectively. The total score ranged from 0 to 100.The higher the score, the greater the psychology resilience the subjects, the Cronbach's $\alpha$ values was 0.91. In our current study, the Cronbach's $\alpha$ values of CD-RISC was 0.804 showing high reliability.

(3) Social Support Rating Scale (SSRS)

The SSRS developed by Xiao [9] and used to evaluate social support, containing 10 items and three dimensions, respectively is the objective support dimension, the subjective supportdimension and the support utilization dimension. The total score is 66 . Scale with $<22$ means low social support degree, among $23 \sim 44$ means average social support degree, and $>45$ means high social support degree. The questionnaire was of great reliability, the Cronbach's $\alpha$ values was 0.89 0.94. In this study, the Cronbach's $\alpha$ values of SSRS was 0.818.

(4) Stroke self-efficacy questionnaire (SSEQ)

The SSEQ developed by American economist Jones et al. [10] and was translated in China by Li [11], containing 11 items and two dimensions, the daily living dimension and Self-managemen dimension, respectively. The score ranges from 0 to 110. The higher the scores, the better the level of rehabilitation self-efficacy of the subjects. The Cronbach's $\alpha$ values was 0.969. In current study, the Cronbach's $\alpha$ values of SSEQ was 0.869.

\subsubsection{Methods of investigation}

Before the formal investigation, explain the purpose and significance of this research have been illustrated to the department director and department head nurse of the hospital to obtain their consent and support. The investigators selected patients with convalescent stroke and gave them one-to-one guidance to explain the 
significance of the study and precautions. After signing the informed consent, the patients took about 15 to 20 minutes to fill the questionnaire. Then, the questionnaire is collected on the spot and the quality of its contents is checked to ensure data accuracy. A total of 300 questionnaires were distributed, and 288 surveys were included in the data analysis after excluding questionnaires with invalid data. The recovery rate was $96 \%$.

\subsubsection{Data analysis}

In this study, SPSS 19.0 software was used for data analysis. Mean and standard deviation (SD) were used to describe the quantitative data and the qualitative data was represented by case number and percentage description. Spearman correlation test was used to discuss the relationship among social support, self-efficacy and psychological resilience and the mediating effect of self-efficacy which was also verified by using AMOS21.0 software.

\section{Results}

\subsection{General information}

The general demographic data of the patients were gender, age, marital status, educational level, residence, work status, medical expenses, type of stroke, time of stroke, stroke severity (Table 1).

Table 1. General demographic data $(\mathrm{N}=\mathbf{2 8 8})$

\begin{tabular}{|c|c|c|c|}
\hline Items & Group & Frequency $(n)$ & Proportion(\%) \\
\hline \multirow[t]{2}{*}{ Gender } & Man & 165 & $57.3 \%$ \\
\hline & Woman & 123 & $42.7 \%$ \\
\hline \multirow[t]{3}{*}{ Age } & $<45$ & 50 & $17.4 \%$ \\
\hline & $45 \sim 59$ & 93 & $32.3 \%$ \\
\hline & $>59$ & 145 & $50.3 \%$ \\
\hline \multirow[t]{4}{*}{ Marital status } & Unmarried & 13 & $4.5 \%$ \\
\hline & Married & 217 & $75.3 \%$ \\
\hline & Divorce & 32 & $11.1 \%$ \\
\hline & Widowed & 26 & $9.0 \%$ \\
\hline \multirow[t]{4}{*}{ Educational level } & Elementary and below & 22 & $8.0 \%$ \\
\hline & Junior middle school & 55 & $19.1 \%$ \\
\hline & High school & 108 & $37.2 \%$ \\
\hline & College or higher & 103 & $35.8 \%$ \\
\hline \multirow[t]{2}{*}{ Residence } & City & 243 & $84.4 \%$ \\
\hline & Countryside & 45 & $15.6 \%$ \\
\hline \multirow[t]{3}{*}{ Work status } & On the job & 90 & $31.3 \%$ \\
\hline & Retire & 122 & $42.4 \%$ \\
\hline & Jobless & 76 & $26.4 \%$ \\
\hline \multirow[t]{3}{*}{ Medical expenses } & Self-paying & 15 & $5.2 \%$ \\
\hline & public expense & 25 & $8.7 \%$ \\
\hline & Health insurance & 248 & $86.1 \%$ \\
\hline \multirow[t]{3}{*}{ Type of stroke } & Cerebral infarction & 196 & $68.1 \%$ \\
\hline & Cerebral hemorrhage & 83 & $28.8 \%$ \\
\hline & Mixed & 9 & $3.1 \%$ \\
\hline \multirow[t]{3}{*}{ Time of stroke } & 2 weeks $\sim 1$ months & 85 & $29.5 \%$ \\
\hline & 1 months $\sim 3$ months & 73 & $25.3 \%$ \\
\hline & 3 months $\sim 6$ months & 130 & $45.1 \%$ \\
\hline \multirow[t]{4}{*}{ Stroke severity } & Complete disability & 15 & $5.2 \%$ \\
\hline & Severe disability & 116 & $40.3 \%$ \\
\hline & Moderate disability & 123 & $42.7 \%$ \\
\hline & No disability & 34 & $11.8 \%$ \\
\hline
\end{tabular}




\subsection{Scores of social support, self-efficacy and psychological resilience in patients with convalescent stroke}

The results of this survey show that the total score of social support for patients with convalescent stroke is (34.25 \pm 5.95$)$, the total score of self-efficacy is $(60.28 \pm 13.11)$, and the total score of psychological resilience is (61.90土13.84), which are show in Table 2.

Table 2. Scores of social support, self-efficacy and psychological resilience(n=288,M \pm SD)

\begin{tabular}{ccc}
\hline Variables & Each dimension & Score $(\mathrm{M} \pm \mathrm{D})$ \\
\hline Social support & Total score & $34.25 \pm 5.95$ \\
& Subjective support & $8.97 \pm 2.57$ \\
& Objective support & $17.82 \pm 4.12$ \\
Self-efficacy & Support utilization & $7.46 \pm 2.03$ \\
& Total score & $60.28 \pm 13.11$ \\
Psychological resilience & Daily living & $35.04 \pm 8.94$ \\
& Self-management & $25.20 \pm 7.95$ \\
& Total score & $61.90 \pm 13.84$ \\
& Tenacity & $30.51 \pm 9.94$ \\
\hline
\end{tabular}

\subsection{Correlation analysis of social support, self-efficacy and psychological resilience in patients with convalescent stroke}

Shown in Table 3, the results of Spearman correlation analysis showed that there was a significant positive correlation between the score of psychological resilience, social support, and self-efficacy in patients with stroke during recovery.

Table 3. Correlation between social support, self-efficacy, and psychological resilience (r)

\begin{tabular}{|c|c|c|c|c|}
\hline variables & Psychological resilience & Tenacity & Self-improvement & Optimistic \\
\hline Social support & $0.778^{* *}$ & $0.650^{* *}$ & $0.491^{* *}$ & $0.436^{* *}$ \\
\hline Subjective support & $0.344^{* *}$ & $0.289^{* *}$ & $0.268^{* *}$ & $0.183^{* *}$ \\
\hline Objective support & $0.571^{* *}$ & $0.482^{* *}$ & $0.368^{* *}$ & $0.341^{* *}$ \\
\hline Support utilization & $0.509^{* *}$ & $0.412^{* *}$ & $0.366^{* *}$ & $0.239^{* *}$ \\
\hline Self-efficacy & $0.750^{* *}$ & $0.658^{* *}$ & $0.451^{* *}$ & $0.409^{* *}$ \\
\hline Daily living & $0.463^{* *}$ & $0.381^{* *}$ & $0.353^{* *}$ & $0.309^{* *}$ \\
\hline Self- management & $0.706^{* *}$ & $0.626^{* *}$ & $0.383^{* *}$ & $0.349^{* *}$ \\
\hline
\end{tabular}

$P^{* *}<0.01$

\subsection{The mediating effect of self-efficacy between social support and Psychological resilience in patients with convalescent stroke}

In this study, Amose 22.0 statistical analysis software was used to test the structural equation model, and the maximum likelihood method was used to estimate the model, and shown in Figure 1. From the perspective of the final model fitting indicators, GFI, AGFI, RMSEA and other major fitting evaluation indexes have reached the standard, the structural equation model has a good fitting degree (Table 4).

Then Bias-corrected percentile Bootstrap method was used to test the significance of the self-efficacy mediation effect which validates the incomplete mediating effect of self-efficacy between social support and psychological resilience. The normalized estimated path coefficient of social support for self-efficacy is $1.80(P<0.05)$ and for psychological resilience is $0.85(P<0.05)$. The normalized estimated path coefficient of self-efficacy for psychological resilience is $0.54(P<0.05)$. Therefore, the mediation effect of self-efficacy is $0.97(1.8 * 0.54)$, and 
the mediation ratio is $53.30 \%(0.97 /(0.97+0.85) * 100)$ (Table 5 and Table 6$)$.

Table 4. Goodness-of-fit statistics for model

\begin{tabular}{cccccccc}
\hline Fitting index & $\mathrm{X}^{2} / \mathrm{df}$ & GFI & AGFI & NFI & TLI & CFI & RMSEA \\
\hline Reference value & $<5$ & $>0.9$ & $>0.9$ & $>0.8$ & $>0.8$ & $>0.9$ & $<0.08$ \\
Model & 1.66 & 0.98 & 0.95 & 0.96 & 0.97 & 0.98 & 0.05 \\
\hline
\end{tabular}

$\mathrm{X}^{2} / \mathrm{df}$,Chi-square to degrees of freedom ratio; GFI, goodness-of-fit index; AGFI: adusted goodness-of-fit index; NFI, normed fit index; TLI, Tucker-Lewis index; CFI, comparative fit index; RMSEA indicates root mean square error of approximation.

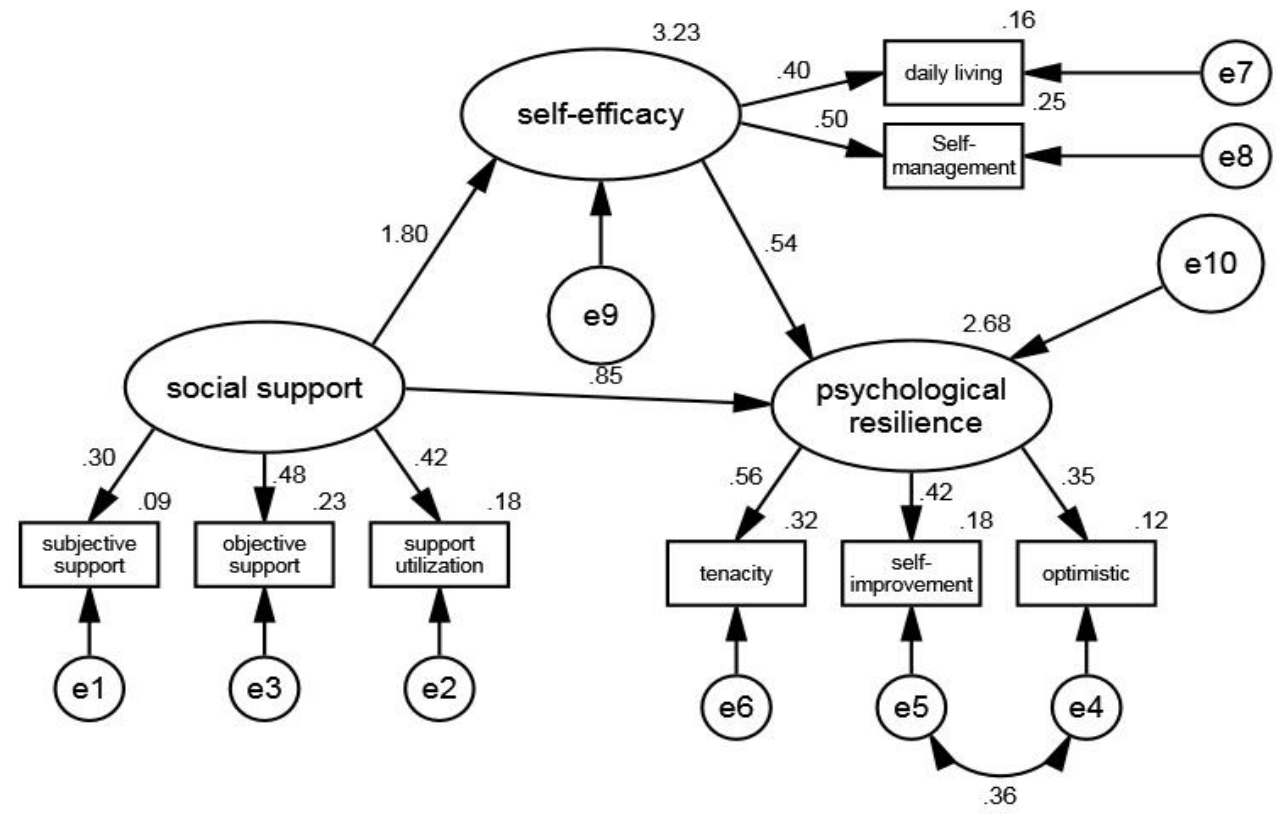

Fig. 1 The mediating effect of self-efficacy between social support and psychological resilience

Table 5. Model path coefficient estimation results

\begin{tabular}{ccccc}
\hline Item & Estimate & SE & CR & P \\
\hline Self-efficacy $\leftarrow$ Social support & 7.55 & 1.66 & 4.54 & $<0.01$ \\
Psychological resilience $\leftarrow$ Self-efficacy & 0.16 & 0.04 & 4.06 & $<0.01$ \\
Psychological resilience $\leftarrow$ Social support & 1.02 & 0.19 & 5.31 & $<0.01$ \\
\hline
\end{tabular}

Estimate,Normalized path coefficient estimate; SE,Estimated Standard Error; CR,Critical ratio

Table 6. Effect of social support, self-efficacy and psychological resilience

\begin{tabular}{ccccc}
\hline Independent variable & Dependent variable & Direct effect & Indirect effect & Total effect \\
\hline Social support & Psychological resilience & 0.85 & 0.97 & 1.82 \\
Self-efficacy & Psychological resilience & 0.54 & 0.00 & 0.54 \\
Social support & Self-efficacy & 1.80 & 0.00 & 1.80 \\
\hline
\end{tabular}

\section{Discussion}

\subsection{Status of social support, self-efficacy and psychological resilience in patients with convalescent stroke}

In this study, the score of social support in patients with convalescent stroke is at a medium level, which is 
lower than that of Zhang [12] in the investigation of patients with community cerebral infarction. Stroke is a chronic disease. A series of sequelae after stroke influences, lead to a decline in patients' self-care ability and social ability. Some patients have poor psychological endurance, causing a variety of negative psychology in patients easily and thus affect access to social support.

This study shows that the self-efficacy score of patients with convalescent stroke is lower than that of Liang [13] using the same scale to investigate the self-efficacy of young and middle-aged stroke. The possible reason is that the subjects of this study are mainly middle-aged and elderly patients. The older the patient who encouraged more severe physical abrasion, lower ability of cooperating effectively, which seriously affect the compliance of treatment and confidence in rehabilitation exercise.

The level of Psychological resilience of patients with convalescent stroke in this study is lower than that of $\mathrm{Yu}$ [14] in the general population of China, and lower than that of the general population of the United States [15], which indicates that the level of psychological resilience of patients with convalescent stroke is relatively low. Psychological resilience is a protective factor for individual health, which can intuitively reflect the dynamic response of mental activities of stroke patients [16]. Therefore, our medical staff should pay attention to the patient's psychological adverse emotions while taking care of the patient's disease, and take appropriate measures to reduce the patient's psychological burden to promote the physical and mental health of patients.

\subsection{The relationship between social support, self-efficacy and psychological resilience in patients with convalescent stroke}

The results of this study show that social support and psychological resilience of patients with convalescent stroke are positively correlated. That is, the higher the level of social support, the better the psychological resilience of patients, which is consistent with the findings of $\mathrm{Xu}$ [17]. Social support, as an important buffer system for individual stress, can alleviate the negative effects of external influences on the patient's psychological resilience. Hu [18] shows that the more social support patient get, the greater the availability of social support, which greatly improves the patient's compliance with the doctor's order, and is beneficial to the patient's disease recovery.

This study show that there is a positive correlation between self-efficacy and psychological resilience in patients with convalescent stroke. That is, the self-efficacy level can significantly affect the patient's psychological resilience score, and the patients with higher self-efficacy level have higher psychological resilience score. The results are similar to the those of Zhang [19]. Studies have shown that the self-efficacy level of stroke patients is related to post-stroke depression. Improving the patient's self-efficacy can prevent the occurrence of depression [20]. Self-efficacy plays an important role in the process of self-regulation, which can improve the patient's coping ability and frustration tolerance and improve their psychological resilience.

\subsection{Mediating effects of self-efficacy in patients with convalescent stroke}

Structural equation model shows that self-efficacy plays a mediating role between social support and psychological resilience, that is, the positive effect of social support on psychological resilience increases with the increase of self-efficacy. The proportion of self-efficacy mediation effect was $53.30 \%$. Social support not only has a direct predictive effect on psychological resilience $(P<0.01)$, but also has an indirect prediction effect on psychological resilience by affecting the self-efficacy level $(P<0.01)$. It can be seen that one of the key factors that improve the impact of social support on the level of psychological resilience of patients with convalescent stroke is having a high level of self-efficacy. Patients with a high self-efficacy are more able to actively seek social support from various sources, actively face the pressure caused by traumatic events to improve their level of psychological resilience. Studies have shown that self-efficacy plays a regulating role in the process of social support affecting anxiety, and with the self-efficacy improves, the negative regulating effect of social support on anxiety is enhanced [21]. Li [22] found that elderly patients with knee osteoarthritis, social support and self-efficacy can be used as influencing factors of psychological resilience, and there is a positive relationship between the two. This suggests our medical staff not only to focus on building a social support system for patients with stroke during recovery, but also to enhance the level of self-efficacy of patients while improving social support levels, fully increasing patients' confidence in facing the disease, reduce the anxiety, sadness and other bad psychology, and improve the level of psychological resilience. 


\section{References}

[1] Lindsay MP, Norrving B, Sacco RL, et al. (2019). World Stroke Organization (WSO): Global Stroke Fact Sheet 2019 [J]. International Journal of Stroke, 14(8): 806-817.

[2] Zhao Q, Shi GF. (2018). Research progress of rehabilitation of patients with limb movement disorder after stroke [J]. Chinese Journal of Nursing Education, 15(11): 873-877.

[3] Mou Y, Deng BQ. (2018). Analysis of psychological health status and related factors in post-stroke hemiplegia patients [J]. Anhui Medical and Pharmaceutical Journal, 22(11): 2127-2130.

[4] Huisman M, Klokgieters SS, Beekman ATF. (2017). Successful ageing, depression and resilience research; a call for a priori approaches to investigations of resilience [J]. Epidemiology and Psychiatric Sciences, 26(06): 574-578.

[5] Lo SH, Chang AM, Chau JP. (2016). Translation and Validation of a Chinese Version of the Stroke Self-Efficacy Questionnaire in Community-Dwelling Stroke Survivors [J]. Topics in Stroke Rehabilitation, 23(3): 163-169.

[6] The Fourth National Cerebrovascular Disease Conference. Main points for diagnosis of various cerebrovascular diseases [J]. Chinese Journal of Neurology. 1996, 29(6): 60-61.

[7] Connor KM, Davidson JRT. (2003). Development of a new resilience scale: The Connor-Davidson Resilience Scale (CD-RISC) [J]. Depression and Anxiety, 18(2): 76-82.

[8] Yu XN, Zhang JX. (2007). Comparison of Self-Resilience Scale and Oonnor-Davidson Resilience Scale [J]. Journal of Psychological Science, 30(5): 1169-1171.

[9] Xiao SY. (1994). The Theoretical Basis and Research Application of Social Support Rating Scale [J]. Journal of Clinical Psychiatry, 1994(02): 98-100.

[10] Jones F, Partridge C, Reid F. (2008). The Stroke Self-Efficacy Questionnaire: measuring individual confidence in functional performance after stroke [J]. Journal of Clinical Nursing, 17(7b): 244-252.

[11] Li HY, Fang L, Bi RX, et al. (2015). The reliability and validity of the Chinese Version of Stroke Self-Efficacy Questionnaire [J]. Chinese Journal of Nursing, 50(07): 790-794.

[12] Zhang BH, Li ShH. (2018). Correlation between self-care ability and social support in patients with community cerebral infarction [J]. Chinese Practice Nursing, 16(09):1127-1129.

[13] Liang LL, Xu MY, Lin BL, et al. (2018). Rehabilitation self-efficacy and influencing factors in young and middle-aged patients with stroke [J]. Guangdong Medical Journal, 39(10): 1535-1538.

[14] Yu XN, Zhang JX. (2005). Resilience-The psychological mechanisms for recovering and growing under pressure [J]. Advances in Psychological Science, 2005(05): 658-665.

[15] Connor KM, Davidson JRT. (2003). Development of a new resilience scale: The Connor-Davidson Resilience Scale (CD-RISC) [J]. Depression and Anxiety, 18(2): 76-82.

[16] Bo T, Zhao QQ, et al. (2019). The Correlation Study among Mental Resilience, Anxiety and Depression in Stroke patients [J]. Journal of Qilu Nursing, 25(03): 36-39.

[17] Xu J, Sun WJ, Tang QS. (2020). Correlation between social support and psychological resilience and depression in patients with stroke [J]. Journal of Inner Mongolia Medical University, 36(04): 16-19.

[18] $\mathrm{Hu} \mathrm{M}, \mathrm{Hu} \mathrm{XJ}, \mathrm{Wu} \mathrm{CH}$, et al. (2020). Feasibility analysis of improving social support of mild craniocerebral trauma patients to improve their quality of life [J]. Chinese Journal of General Practice. 2020(01):139-141.

[19] Zhang M, Liu ZH, Li JJ, et al. (2019). The level of psychological resilience in first-attack stroke patients and its in fluencing factors [J]. Nursing Journal of Chinese People's Liberation Army, 36(04): 16-19.

[20] Volz M, Möbus J, Letsch C, et al. (2016). The influence of early depressive symptoms, social support and decreasing self-efficacy on depression 6 months post-stroke [J]. Journal of Affective Disorders, 206: 252-255.

[21] Wang YJ, Zhou LL. (2019). Regulation of self-efficacy between social support and anxiety for primipara [J]. Journal of Nursing and Rehabilitation, 18(12):13-16.

[22] Li SM. (2019). Study on resilience level and intervention of elderly patients with knee osteoarthritis in nursing institution [D]: Shanxi University of Traditional Chinese Medicine. 